\title{
Study on Interfacial Science and Rheology
}

\author{
Hitesh Rawate $^{1}$, A K Goswami ${ }^{2}$, \\ Department of Chemical Engineering ${ }^{1,2}$, University Institute of Chemical Technology $y^{1,2}$ \\ Email: hitesh.hr238@gmail ${ }^{1}$, goswamiakg1@gmail.com ${ }^{2}$
}

\begin{abstract}
The study focuses on the interfacial science and the rheology of the fluids at the interface. The basics of interface and interfacial properties are discussed. The term rheology is explained and the measurement methods of rheology are elaborated to calculate the interfacial properties. The future application of this study in different fields is explained. Different types of literature are discussed in the field of chemical engineering and biology. The applications like Bio-Interface, Nano-medicine are studied by the literature.
\end{abstract}

Index Terms- Interface, Rheology, Bio-interface, Nano-medicine, etc.

\section{INTRODUCTION TO INTERFACIAL SCIENCE AND RHEOLOGY}

When the two immiscible fluids contacted each other's then they share a common physical region of contact called as the interface. This interface is nothing but the physical region separating the two fluids, where the fluid may be gases or liquids. This interface separates the immiscible fluids physically. Interfaces have somewhat different characteristics than that of both phases and due to which the effective separation can be characterized by these interfacial properties. The variation of characteristics at the interface is observed because; at the interface, both the phases have a common physical region in contact but with different physical properties. As the phase separation is totally dependent on these interfacial properties formed by the two phases, the analysis became very important for various processes such as Emulsification, Foam formation, Desalting operation, Extraction, etc. Therefore, the different fields like chemical technology, biosciences, oil industries, surfactant and detergent research, etc. knowledge of the interface and its properties are essential. In Food formulation, Personal care product, Petrochemical units it is necessary to make a control over the size distribution of emulsion and foams. Also, the control over the stability of these foams size and emulsion molecules make a great advantage on system efficiency.

Rheology is one of the methods available to characterize these interfacial properties. Rheology is the study of the fluid undergoing the deformation. In rheology, we apply stress to deform the fluid and the respective strain is measured for the given deformation. This dependency of stress to the deformation or deformation rate is used to characterize the interfacial properties. This approach is mostly used to measure the viscoelastic properties of the fluid. The 2-dimensional (Surface) rheology is used to study the interfaces. The purpose of surface rheology is to find the effect of any foreign molecule at the interface on the flow of the liquid. The flow can be affected by the interface as the stresses at the interface and the bulk on the motion are different.

Measurement of Rheological properties can be done in 3 stages;
1. Stress
2. Strain
3. Stress to Strain ratio

The stress can be identified by the force applied for a given area and the strain is the degree of deformation for respective stress. The ratio of these stress and strain is used for the elastic modulus calculation for solids and viscosity calculation for fluids. The most common problem in fluid operations is that the number of materials comes under both liquid and solid aspects and these results in the variation in the properties like elastic moduli and viscosity.

It is very much important to differentiate stress and strain as many of us get confused between them. The strain is the result of Stress applied and the value of the strain deformation is depending on the amount of stress applied. The magnitude and the direction are very much important in the stress analysis. The magnitude of stress is calculated using the force applied (for example, if you stand on 1000 vertical needled it won't be penetrated inside the foot but if you stand on the single vertical needle you definitely will. This is because of the force by your weight is same in both cases but the area covered by a needle in both cases is different and result in more stress on a single needle than that of 1000 needles). The direction of the stress applied defines the type of stress i.e. tensile, shear, etc. and the unit of the stress is a simple to force per unit area (If the force is parallel to surface then it is a shear stress and if it is perpendicular or normal to surface then it is called normal stress or simple pressure, and also if the stress is applied for both the side in the opposite direction then it is the tensile stress and vice versa is called compressive stress). The strain rheology is purely by geometry means and calculated by the amount of deformation from the reference position. The strain is the unit less quantity and most of the problems in the 
International Journal of Research in Advent Technology, Vol.7, No.4S, April 2019

\author{
E-ISSN: 2321-9637 \\ Available online at www.ijrat.org
}

rheology are associated with strain deformation. Sometimes it is difficult to calculate the area and the location where force is applied due to irregular shapes of the sample.

Using the ratio of stress to stain we can compute the elastic modulus which allows the prediction of material deformation for a given force. Similarly, the viscosity is also provided using the stress to strain ratio and it will provide the resistances and flow velocities for a given fluid. In general, stress is directly proportional to stain i.e. if you apply more stress more deformation you observe or vice a versa. On the application of the stress, if the strain reaches a limit then it is viscous but if it increases continuously then it is elastic in nature. The perfectly elastic material will deform continuously till its final stain state and regain its original shape after removal of the stress. For elastic materials, the stress-strain ratio is always constant and the work applied by the stress is stored in itself and used to regain its original shape after removal of stress. Simply elasticity is the ability to regain its original shape after removal of stress. Depending on the type of stress applied, the different type of elastic modulus is available (for shear stressshear modulus is calculated and for tensile or compressive stress Young's modulus is calculated). The ratio of stress to strain to the strain rate is used for the measurement of viscosity. Most of the biological material possesses both the elastic as well as viscous properties. The strain magnitude and strain rate are important to define the shear modulus and viscosity of the fluid. Use of oscillatory deformation with various frequencies gives differentiation of elastic from viscous effects. The results are loss or storage modulus also called as viscous moduli or elastic moduli respectively.

\section{APLICATIONS OF INTERFACIAL SCIENCE AND RHEOLOGY}

There are many fields and branches of science where the study of the interface is beneficial to understand the surface behavior of molecules. This study helps to determine interfacial molecular activities and viscoelastic properties of molecules at the interface. In most of the system such as foaming, emulsion, soap manufacturing, desalting operation, enhanced oil recovery, the surface-active molecules are involved. In such a case, the large interfacial area is exposed to contact, and that's why the study of interfaces provides an advantage to these applications. Wherever the surface-active molecule involves, it adsorbs at the interface resulting in decreasing the interfacial tension and increasing the interfacial pressure. In the case of emulsions, the stability of the emulsion is the main problem to be focused. This stability is achieved by entering the surface-active molecule in the system. Also, in some applications like wastewater treatment and enhanced oil recovery, we have to separate the heavy liquid or oil from water that's why the stability is unwanted. This stability and instability can be achieved by the knowledge of the interfaces.

In the subjects like Reaction Engineering \& Mass Transfer and the processes like Catalysis, Extraction \& membrane separation, it is necessary to study the interfaces because the most of the physiochemical process, as well as the reactions, occurs at the interface or the surfaces. The surface or interface of any solid or liquid have quite unique and different properties than that of the bulk material. The simple example of these phenomena is water, where the denser materials float on the surface of the water because of the high surface tension at the air-water interface. Similarly, in chemistry, we deal with various colloids. Where colloids are the complex material and the behavior of the colloidal system can be found by the interactions between phases or molecules at their interface. Some common examples of these colloids are Emulsions, Dispersion, Foams, and Aerosols etc. The new challenges are coming from the research and development of the solid/liquid interface at the molecular level. As the physical properties of both the faces involved in solid/liquid interfaces, it is difficult to correlate the bulk properties of these both phases at the interface.

The advances in the medical field have affected human life to be healthier. More research in a few decades has made the number of disease curable. These researches have mainly based on the application of bio-interfaces. The bio-interface is the contact region shared by the bio-molecule, cell, tissue and any other organic/inorganic matter. In the field of diagnostic, medicine and biology it is important to study the behavior of the molecule of any vaccine or drug at the bio-interfaces. The complex structure and its properties are difficult to understand in biological materials such as cell elongation, cell merging, and drug delivery system by molecule etc. The knowledge of bio-interface makes understanding of these molecular phenomena easier. The extreme scientific challenges forced new researchers to understand these biological surfaces and interfaces in depth to know the actual interaction between different biological systems and thus leads to develop new novel materials and methodologies for a cure. The bio-molecule self-assembly on solid material is very much useful to understand in biological interactions mainly where protein is employed.

The book in the 1950s entitled "An outline of general physiology" by L. Heilbrunn stated that "In an attempt to interpret the machinery of a living cell, it is essential to know something about the mechanical properties of the protoplasm in the cell that is being investigated." Cells have Mechanical, Chemical and Electrical properties and to understand the biology of cells we have to know the knowledge of these all. Numbers of researches are going on the effect of external forces of the cell on the internal 
International Journal of Research in Advent Technology, Vol.7, No.4S, April 2019

\author{
E-ISSN: 2321-9637 \\ Available online at www.ijrat.org
}

force and internal properties. Cell growth, division, differentiation and activation can be affected by external forces; therefore, the knowledge of rheology is important to study this. The topics like soft materials and colloidal suspension in the fields like material science and biology are becoming the future scope for many new researchers. The human body is made up of different components such as cell and tissues. The cell has some viscous and elastic properties and that can be changed by the number of cellular processes within the body. Rheology can be employed to measure these changes in the viscous and elastic properties of cells. The structural formations can be studied using the non-oscillatory techniques and for the flow characteristics, oscillatory ones are used. Sample preparation is one of the most difficult tasks for such analysis. The complex biological systems like the cellular system and intact tissues can be studies using proper experimental setup and rheological techniques.

In the human body, there is a small monolayer of pulmonary surfactant is present at the interface of lungs to reduce the energy in the form of work while breathing. The main application of this lung surfactant is to reduce the liquid/air surface tension. This lung surfactant is made up of complex protein-lipid mixture. Interfacial phenomena play a major role in understanding the pulmonary surfactant interaction with lungs.

Adams, Harley \& Jahnke [1] showed gold surface selective peptide interface is used for the selforganization of the cell. Also, the continuous cell elongation without division is future research for living material interfaces. Tucker, Richter \& Berrie [2] designed a motor protein-based Nano-biodevice. In which they bind the histidine-tagged F1-ATPase motor protein to the gold surface. Yucesoy, Hnilova, Boone, Arnold, Snead \& Tamerler [3] designed antibacterial implant material surface. At bio-Nanointerface, they provide the titanium alloy surface and antimicrobial domain provided by the peptide having anchoring sites. Sullivan \& Topoleski [4] studied the biomaterials employed to cure boned and joints artificially by modifying surface properties to strengthen the wear properties. They have also discussed the different new materials to replace conventional materials like cobalt-chromiummolybdenum in orthopedic operations. Sharma, Payne \& Katti [5] studied the improvisation of nanocomposites using a modifier. Here they used the Nano Clays as a modifier. The molecular dynamic simulation is used to study the effect of the modifier to the nanocomposites. In the next paper by Chelariu, Suditu, Mareci, Bolat, Cimpoesu, Leon \& Curteanu [6] they focused on research related to dental anatomy. The study focuses on the alternative to amalgam-based restorative materials. Mostly the alternative is polymeric composite restoratives because of many properties such as the ability to bond the four measure components of teeth i.e. detin enamel, cementum, and pulp. Ordikhani, Yonghyun, \& Zustiak [7] showed the dependency of bio-medical properties and growth of cancer stem cells. Also, they found that the characteristics of the cancer stem cells can be affected by the interfaces involved during the growth of biomaterials. Kucuk \& Edirisinghe [8] conclude that nanospheres can be produced by the engineered microfluidic system and the surface features of these nanospheres can be affected by the solvent employed and the temperature. Surface roughness is used to overcome the size difference. The properties such as surface area, mechanical strength and chemical and thermal stabilities are the main reason behind the increased use of Carbonbased materials.

Nanotechnology provides future scope for the new researches in the field of biology and medical science. The interfacial study between the bio-molecule and the surrounding is very much useful to predict the biological behavior of nonmaterial. The study of nanotechnology in the field of bio-interfaces is increasing day by day but still many problems are increasing and becoming difficult to resolve such as interface issues like non-specific adsorption. To overcome this problem there is a need to learn the bio-interface of nanoparticles and has to achieve a strong control on them in such a way that we can substitute Nano-bio-particles instead of inorganic particles. The growing research in the field of nanotechnology related to medical improvisation to cure the disease is Nano-medicine. Nano-materials can be employed when the antibacterial and anti-odor functionality on human skin is required. The requirement may be in the form of powder, gel or liquid form of the product.

Lee SE, Liu GL, Kim F \& Lee LP [9] studied the combined effect of Nano-technology and biology. In their research, they found that Nanoparticles can be used to kill tumors \& drug delivery system. These applications can be achieved by non-specific adsorption in which the nanoparticles are stacked to biomolecules covalently. They form a complex 3-dimensional interface. Nel AE, Hoek EMV, Castranova V, Thompson M. and co-workers [10] stated that the nanoparticles do not have any well-defined shape whereas the biomolecules have the detailed and well-defined shape and this shape can be determined by various interaction of intramolecular and intermolecular forces of attraction. The nanoparticles are affected by the nonspecific adsorption. Therefore, for better research and development in medical applications, the biointerface needs to be understood clearly.

\section{CONCLUSIONS}

Interfaces occurs wherever two different fluids involves and this interface plays a major role for separation of these two fluids require especially in 
International Journal of Research in Advent Technology, Vol.7, No.4S, April 2019

E-ISSN: 2321-9637

Available online at www.ijrat.org

case of emulsions and foams. There are number of ways to study these interfaces but in most of the case Rheology is most suitable. The reason behind the suitability if Rheology in interfacial study is that it can measures the properties with even a small deviation in a surface pressure / tension. The use of these interfacial studies and Rheology in the field of chemical engineering and medical science and technology is studied and explained in our study and the future scope and current scenario of medical technology is discussed using the different literature

\section{REFERENCES}

[1] B.L. Adams, M.M. Harley, J.P. Jahnke, and D.N. StratisCullum, JOM, $67 \quad$ (2015). doi:10.1007/s11837-015-1662-7.

[2] J.K. Tucker, M.L. Richter, and C.L. Berrie, JOM (2015). doi:10.1007/s11837-015-1378-8.

[3] D.T. Yucesoy, M. Hnilova, K. Boone, P.M. Arnold, M.L. Snead, and C. Tamerler, JOM 67, 733 (2015).

[4] S.J.L. Sullivan and L.D. Timmie Topoleski, JOM (2015). doi:10.1007/s11837-015-1543-0.

[5] S. Sharma, K.S. Payne, and D.R. Katti, Katti. JOM 67, 733 (2015).

[6] R. Chelariu, G.D. Suditu, D. Mareci, G. Bolat, N. Cimpoesu, F. Leon, and S. Curteanu, JOM 67, 767 (2015). R

[7] F. Ordikhani, K. Yonghyun, and S. Zustiak, JOM (2015). doi:10.1007/s11837-015-1626-y.

[8] I. Kucuk and M. Edirisinghe, JOM 67, 811 (2015) I. Kucuk and M. Edirisinghe, JOM 67, 811 (2015)

[9] Lee SE, Liu GL, Kim F, Lee LP. Remote optical switch for localized and selective control of gene interference. Nano Lett 2009; 9:562-570. [PubMed: 19128006]

[10] Nel AE, Madler L, Velegol D, Xia T, Hoek EMV, Somasundaran P, Klaessig F, Castranova V, Thompson M. Understanding bio-physicochemical interactions at the Nano-bio interface. Nat Mater 2009; 8:543-557. [PubMed: 19525947] 